\title{
Biologia reprodutiva de Sphoeroides testudineus (Linnaeus) (Pisces, Osteichthyes, Tetraodontidae) da gamboa do Baguaçu, Baía de Paranaguá, Paraná, Brasil
}

\author{
Claudia Rocha ${ }^{1,3}$ \\ Luís F. Favaro ${ }^{2}$ \\ Henry L. Spach ${ }^{3}$
}

\begin{abstract}
Reproductive biology of Sphoeroides testudineus (Linneus) (Pisces, Osteichthyes, Tetraodontidae) of the gamboa do Baguaçu, Bay of Paranaguá, State of Paraná, Brazil. The present study seeks to elucidate reproductive aspects of Sphoeroides testudineus (Linnaeus, 1758), Tetraodontidae. Monthly collections were accomplished for one year (November/98-October/99), in the gamboa do Baguaçu, Bay of Paranaguá with a Fyke net. The macroscopic analysis of the gonad stages allowed us to estimate the sexual proportion as 1:1 during the whole period, except in the months of May and October, when the proportions of two females for one male and two males for one female were observed respectively. The analysis of the relative frequency of the monthly gonad stages and the Curve of Maturation aided in the determination of the reproductive period, that occurred from September to January. Microscopic analyses of the female gonads allowed us to characterize four development phases of the ovarian follicle, six stages of ovarian development and the type of spawn, which was parceled. The first maturation for females was estimated to be between $10-11 \mathrm{~cm}$ of length
\end{abstract}

KEY WORDS. Tetraodontidae, puffer fishes, reproduction

Os ecossistemas estuarinos são importantes para reprodução e desenvolvimento de várias espécies de peixes, os quais geralmente possuem alta capacidade de adaptação às variações abióticas características destes ambientes. Os complexos estuarinos da região sul-sudeste do Brasil são caracterizados por apresentarem canais no manguezal, chamados gamboas, responsáveis pelo grande fluxo de matéria orgânica entre continente e estuário, além de abrigarem adultos e formas juvenis de peixes e crustáceos durante seu desenvolvimento.

Segundo DiAs et al. (1998), estudos reprodutivos permitem não só compreender os mecanismos envolvidos na perpetuação das espécies, mas também uma melhor administração pesqueira baseada no uso que os indivíduos de uma espécie fazem de um sistema ou área.

Dentre as diversas espécies estuarinas, Sphoeroides testudineus (Linnaeus, 1758) (baiacu pintado) despertou um grande interesse pela sua abundância em

1) Bolsista de Iniciação Científica PIBIC (CNPq).

2) Departamento de Biologia Celular, Centro Politécnico, Universidade Federal do Paraná. Caixa Postal 19031, 81531-990 Curitiba, Paraná, Brasil.

3) Centro de Estudos do Mar, Universidade Federal do Paraná. Avenida Beira Mar, 83255-000 Pontal do Paraná, Paraná, Brasil. 
sondagens preliminares, pela escassez de literatura e pela sua importância na cadeia trófica (TARgetT 1978). De acordo com Figueiredo \& MENEzes (2000) $S$. testudineus ocorre desde Nova Jersey (EUA) até o estado de Santa Catarina (Brasil).

No Brasil, espécies da família Tetraodontidae possuem baixo valor econômico devido à presença de uma toxina (tetrodotoxina) concentrada na pele, no fígado e nas gônadas. Porém, espécies asiáticas da mesma família possuem alto valor comercial no Japão e seu consumo está condicionado à técnicas de preparo. MATSUMURA (1995) observou que a tetrodotoxina age como um ferormônio feminino, atraindo os machos. Os folículos ovarianos liberados possuem alta concentração de tetrodotoxina na membrana vitelina, a qual induz a liberação dos espermatozóides na água.

Em função da literatura escassa sobre biologia reprodutiva de peixes estuarinos e pela grande importância ecológica desempenhada por S. testudineus, o presente estudo visa um maior conhecimento do seu ciclo reprodutivo, através da caracterização da ovogênese, desenvolvimento ovariano, determinação da época reprodutiva, proporção sexual e tamanho de primeira maturação para fêmeas.

\section{MATERIAL E MÉTODOS}

Foram realizadas coletas mensais no período de novembro de 1998 a novembro de 1999, na gamboa do Baguaçu, Baía de Paranaguá, utilizando-se uma rede fixa tipo "Fyke" ( 30 x 1,5m), com malha de 10 e $8 \mathrm{~mm}$.

Dos 582 exemplares de $S$. testudineus coletados foram registrados dados de comprimentos total $(\mathrm{cm})$ e peso total $(\mathrm{g})$. Posteriormente foi feita uma incisão ventral em todos os espécimes, expondo as gônadas para determinação do sexo e análise macroscópica do desenvolvimento ovariano, baseado na escala de maturidade gonadal para teleósteos (VAZZOLER 1996).

As gônadas foram retiradas, pesadas e 178 destas foram fixadas em Bouin por 18 horas e incluídas em parafina para confecção de lâminas histológicas, as quais foram coradas com Hematoxilina-Eosina. Posteriormente as lâminas histológicas foram analisadas em microscopia de luz. A caracterização das fases ovocitárias, determinação dos estádios de desenvolvimento ovariano e correção da análise macroscópica quando necessário, foram baseadas em VAZZOLER (1996) e DiAS et al. (1998).

Para cada exemplar foi calculado a Relação Gônado-Somático (RGS), definido como (peso da gônada/peso total) x 100, obtendo-se posteriormente a média mensal e a partir de sua variação temporal determinou-se a Curva de Maturação. A determinação do tipo de desova foi baseada na distribuição de freqüência relativa de estádios gonadais e na análise histológica.

O comprimento de primeira maturação sexual $(\mathrm{Cpm})$ para fêmeas foi determinado através da curva que relaciona o ponto médio das classes de comprimento total com a frequência relativa de indivíduos adultos. Para o ajuste da referida curva, utilizou-se a equação:

$$
F r=1-e^{-a L_{S}^{b}}
$$


onde: (Fr) freqüência relativa de indivíduos adultos, (e) base do logaritmo neperiano, (a e b) coeficientes estimados pela transformação das variáveis e (Ls) ponto médio das classes de comprimento. A proporção sexual durante o ciclo reprodutivo, foi testada pelo método do $\mathrm{X}^{2}$, com grau de liberdade 1 e 0,05 de significância ( $\mathrm{X}^{2}$ $<3,840$ ), de acordo com VAZZOLER (1996).

\section{RESULTADOS E DISCUSSÃO}

Através da análise microscópica, foram observadas quatro fases de desenvolvimento do folículo ovariano para $S$. testudineus, os quais estão descritos na tabela I.

Tabela I. Descrição das fases ovocitárias para S. testudineus.

\begin{tabular}{|c|c|}
\hline Fases & Descrição \\
\hline I (ovogônias) & $\begin{array}{l}\text { Células pequenas, em ninhos; o citoplasma é reduzido; o } \\
\text { núcleo é grande com nucléolo único, basófilo e central. }\end{array}$ \\
\hline$\|$ & $\begin{array}{l}\text { Células foliculares pavimentosas envolvem o ovócito e } \\
\text { permanecem desta forma durante todo o processo. O } \\
\text { ovócito apresenta um tamanho maior, o citoplasma se } \\
\text { torna mais volumoso, porém ainda reduzido em relação ao } \\
\text { núcleo, que começa a sofrer fragmentação nucleolar (Fig. } \\
\text { 1A). }\end{array}$ \\
\hline III & $\begin{array}{l}\text { Surgem vesículas citoplasmáticas na periferia do ovócito } \\
\text { (sem afinidade por HE) e a membrana vitelina (envolvendo } \\
\text { o ovócito). O citoplasma é mais volumoso e menos } \\
\text { basófilo. Os nucléolos se encontram periféricos ao núcleo } \\
\text { (Fig. 1B). }\end{array}$ \\
\hline IV & $\begin{array}{l}\text { O ovócito se mostra muito aumentado com citoplasma } \\
\text { preenchido por grânulos de vitelo acidófilos. A membrana } \\
\text { vitelina é bem espessa, com estriaçōes transversais } \\
\text { evidentes (Fig. 1C). }\end{array}$ \\
\hline
\end{tabular}

Folículos vazio (Fig. 1D) e atrésico foram considerados estruturas derivadas do processo de desenvolvimento ovocitário.

VIZZIANO \& BEROIS (1990) dividiram em sete fases o desenvolvimento ovocitário para Macrodon ancylodon (Bloch y Schneider, 1801), sendo que a fase de atresia sofreu ainda subdivisões. Para $S$. testudineus, os folículos atrésicos e vazios foram considerados estruturas derivadas do processo de ovogênese.

CHAVES (1989) demonstrou que a ocorrência de ovócitos com hidratação pré-ovulatória de teleósteos possui uma relação muito mais estreita com o ambiente marinho que com o dulcícola. De acordo com DiAs et al. (1998), a hidratação ocorre pouco antes da desova e ovários com folículos hidratados e folículos vazios são evidências de que a desova ocorre na área estudada. No período analisado, para $S$. testudineus, não foram observados ovócitos em hidratação pré-ovulatória, entretanto a observação de folículos vazios e a presença de indivíduos em diferentes estádios de desenvolvimento gonadal durante o período, indicam que a espécie estudada não só desova no estuário, como também faz uso dos recursos deste ambiente durante todo o ano.

O desenvolvimento ovariano para S. testudineus foi dividido em seis estádios e para cada estádio do desenvolvimento foi calculado o IGS médio (Tab. II). 

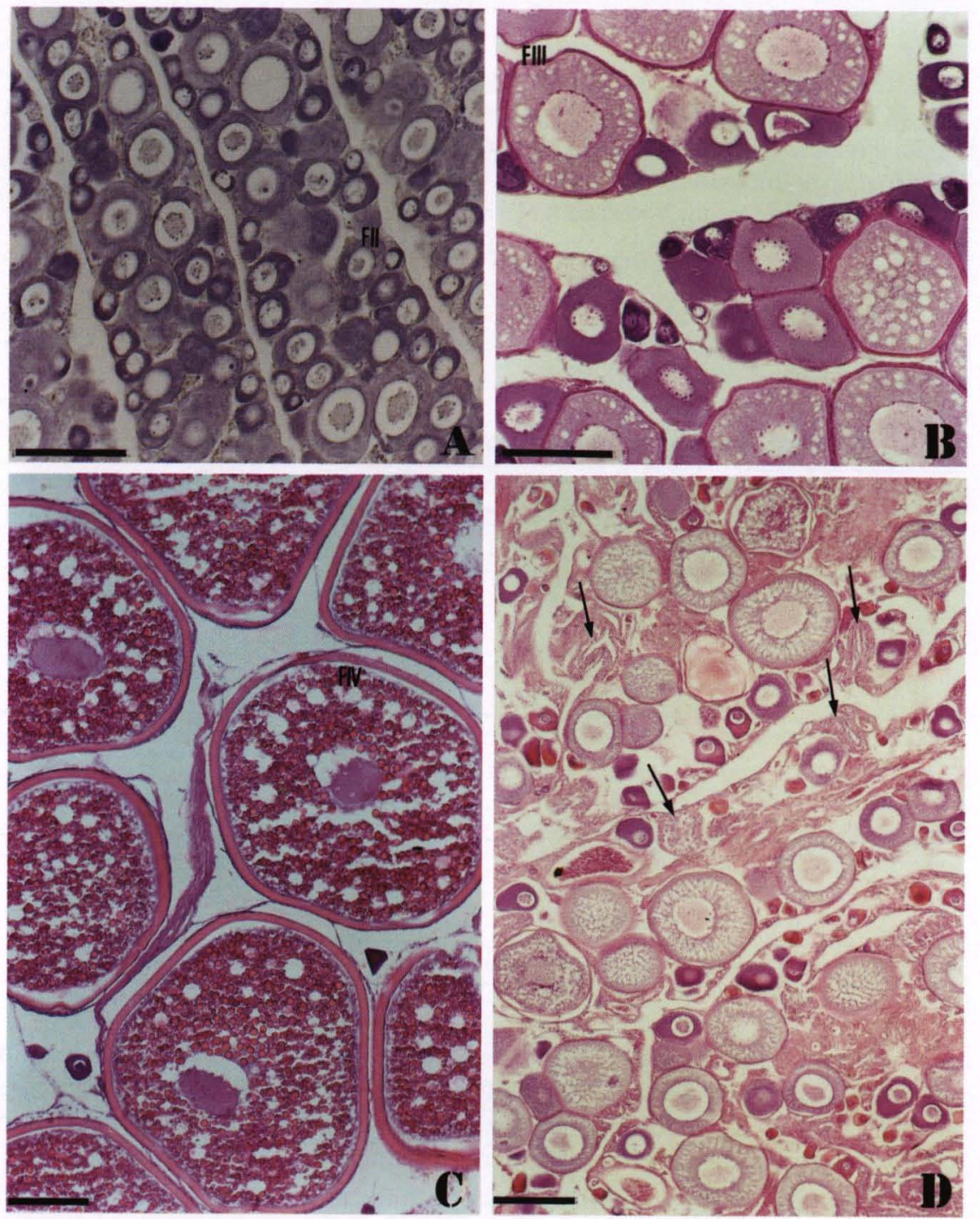

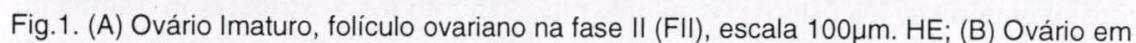

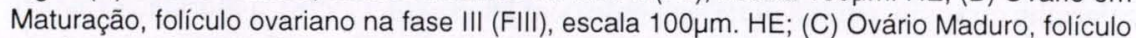

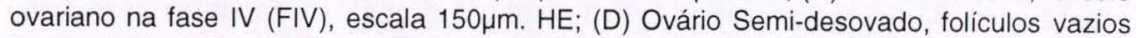
(seta), escala $150 \mu \mathrm{m}$. HE.

De acordo com VAZzolER (1996), a utilização de um indicador quantitativo do período reprodutivo, como o RGS, é importante para contrabalançar a subjetividade dos dados sobre estádio de maturidade baseados em análises macroscópicas das gônadas. Em função desta subjetividade, o cálculo do RGS médio de cada estádio de desenvolvimento gonadal pode auxiliar a classificação macroscópica. 
Tabela II. Estádios do desenvolvimento ovariano.

\begin{tabular}{lcl}
\hline \multicolumn{1}{c}{ Estádios } & RGS Médio & \multicolumn{1}{c}{ Características histológicas } \\
\hline Imaturo (A) & 0,310 & $\begin{array}{c}\text { Ovogônias e ovócitos na fase II em lamelas organizadas } \\
\text { (Fig.1A) } \\
\text { Ovogônias e ovócitos nas fases II e III (Fig. 1B) }\end{array}$ \\
$\begin{array}{l}\text { Maturação (B) } \\
\text { Maduro (C) }\end{array}$ & $\begin{array}{l}1,033 \\
\text { Semi-desovados (SD) }\end{array}$ & $\begin{array}{l}\text { Predomínio de ovócitos na fase IV (Fig. 1C) } \\
\text { Desovado (D) }\end{array}$ \\
$\begin{array}{ll}\text { Abundância de ovócitos na fase III e folículos vazios (Fig. 1D) } \\
\text { Ovócitos na fase II, poucos na fase III e folículos vazios em } \\
\text { lamelas desorganizadas } \\
\text { Ovogônias e ovócitos na fase II, em lamelas mais organizadas } \\
\text { que no estádio anterior. Ausência de folículos vazios }\end{array}$ \\
\hline
\end{tabular}

O período reprodutivo foi baseado na presença de ovários maduros e semi-desovados, compreendendo os meses de setembro a janeiro. Foram encontrados indivíduos maduros nos meses de setembro, outubro, novembro e dezembro.

A observação microscópica de ovários semi-desovados e suas freqüências mensais, permitiram inferir que a desova é do tipo parcelada, iniciando-se no mês de outubro e com duração de aproximadamente quatro meses. Após o período de desova, indivíduos em maturação foram observados novamente a partir de abril. A Curva de Maturação permite observar um longo período de recuperação gonadal, podendo durar até cinco meses (janeiro a maio). O pico de recrutamento de indivíduos jovens ocorre em março, quando foi calculado o menor RGS médio do período e observada a maior frequiência de indivíduos imaturos (Figs 2-3).

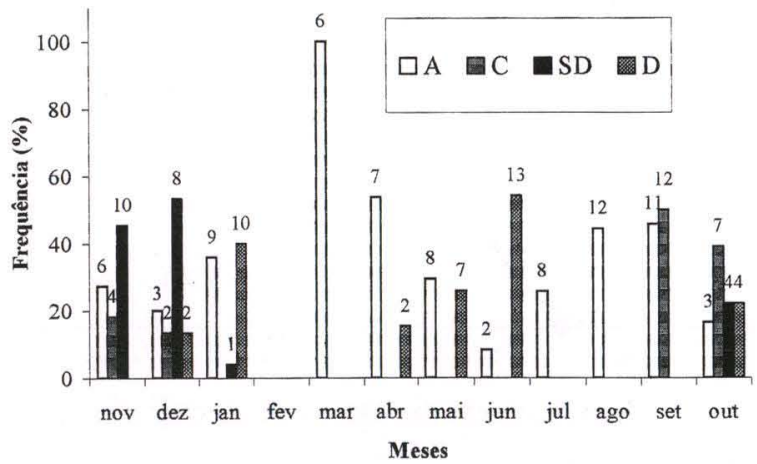

Fig. 2. Freqüência Relativa dos Estádios de Desenvolvimento Gonadal. (A) Imaturo, (C) maduro, (SD) semi-desovado, (D) desovado, (R) recuperado. Os números localizados em cima das barras, correspondem ao número de indivíduos.

A primeira maturação gonadal corresponde ao tamanho em que $50 \%$ dos indivíduos da população são classificados como adultos. Para fêmeas da referida espécie este fato tem início quando os indivíduos atingem o comprimento médio de 10,8cm (Fig. 4). A equação matemática da transformação linear da curva de maturação foi: $\mathrm{Y}=7,1634 \mathrm{X}-17,414\left(\mathrm{R}^{2}=0,9726\right)$, sendo a curva de primeira maturação ajustada pela equação: $\mathrm{fr}=1-\mathrm{e}^{-2,7365 \times 10-8 \mathrm{Lt} 7,1634}$. 


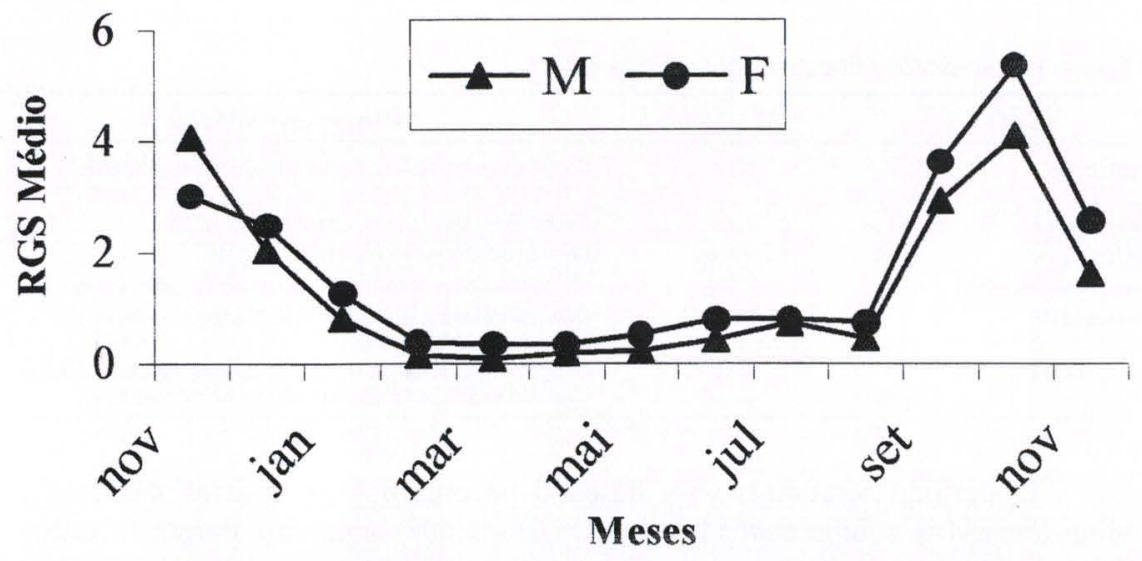

Fig. 3. Curva de Maturação para Machos (M) e Fêmeas (F).

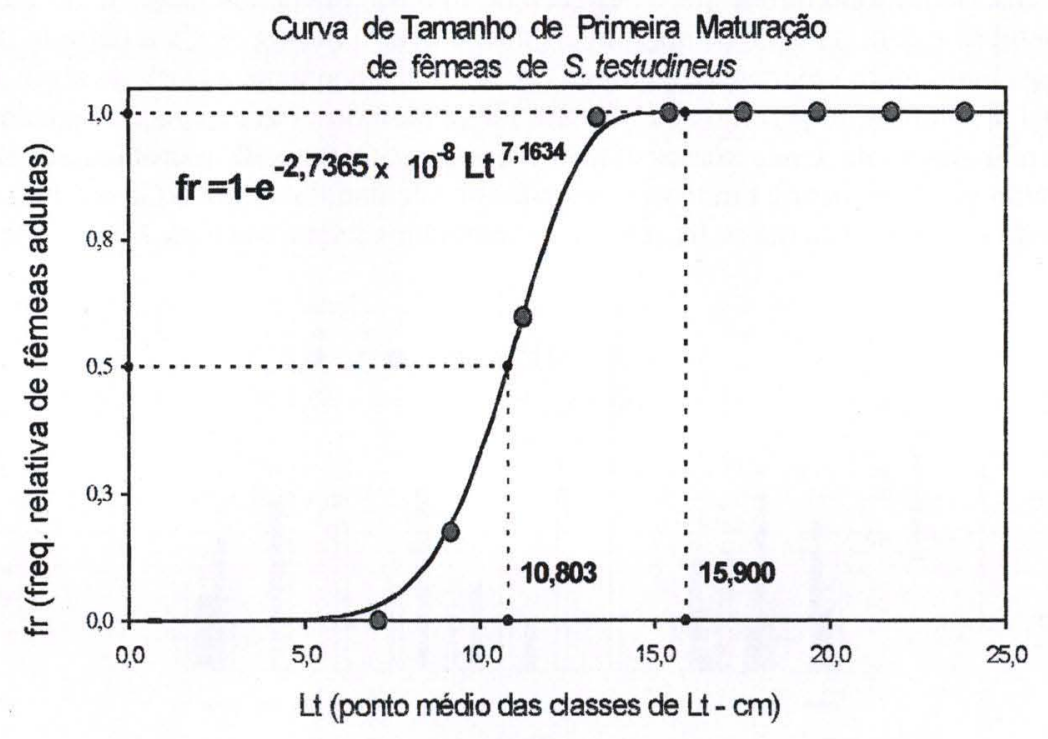

Fig. 4. Comprimento de primeira maturação para fêmeas de Sphoeroides testudineus.

A proporção sexual entre machos e fêmeas apresentou valores significativos apenas para os meses de outubro (dois machos para cada fêmea) e maio (duas fêmeas para cada macho) (Tab. III). Relacionando a Curva de Maturação com os resultados da proporção sexual, constata-se que a proporção de dois machos para uma fêmea coincide com o início da desova. 
Tabela III. Freqüência Absoluta de Machos e Fêmeas e $X^{2}(g l=1)$.

\begin{tabular}{lccc}
\hline Meses & Macho & Fêmea & $X^{2}$ \\
\hline Nov/1998 & 23 & 22 & 0,022 \\
Dez/1998 & 24 & 15 & 2,077 \\
Jan/1999 & 20 & 25 & 0,555 \\
Fev/1999 & 13 & 17 & 0,533 \\
Mar/1999 & 6 & 6 & $1,000^{2}$ \\
Abr/1999 & 17 & 13 & 0,533 \\
Mai/1999 & 13 & 27 & $4,900^{*}$ \\
Jun/1999 & 28 & 24 & 0,308 \\
Jul/1999 & 20 & 31 & 2,370 \\
Ago/1999 & 23 & 27 & 0,320 \\
Set/1999 & 26 & 24 & 0,080 \\
Out/1999 & 34 & 18 & $4,920^{*}$ \\
Nov/1999 & 19 & 10 & 2,790 \\
\hline
\end{tabular}

* Significativo ao nível de $5 \%$.

\section{REFERÊNCIAS BIBLIOGRÁFICAS}

Chaves, P.T.C. 1989. Hidratação pré-ovulatória em peixes: Um caráter de origem marinha? Revta bras. Zool. 6 (3): 463-472.

Dias, J.F, E. Peres-Rio; P.T.C. Chaves; C.L.D.B. Rossi-Wongtschowski. 1998. Análise Macroscópica dos Ovários de Teleósteos: Problemas de Classificação e Recomendações de Procedimentos. Rev. Brasil. Biol. 58 (1): 55-69.

Figueiredo, J.L. \& N.A. Menezes. 2000. Manual de Peixes Marinhos do Sudeste do Brasil. VI.

Teleostei (5). São Paulo, Museu de Zoologia, Universidade de São Paulo, 116p.

Matsumura, K. 1995. Tetrodotoxin as a Pheromone. Nature 378: 563-564.

TARgetT, T.E. 1978. Food Resource Partitioning by the Pufferfisches Sphoeroides spengleri and $S$. testudineus from Biscyne Bay, Florida. Mar. Biol. 49: 83-91.

Vazzoler, A.E.A.M. 1996. Biologia Reprodutiva de Peixes Teleósteos: Teoria e Prática. Maringá, Ed. Univ. Estadual de Maringá, 169p.

Vizziano, D \& N. Berois. 1990. Histología des Ovario de Macrodon ancylodon (Bloch y Schneider,1801) (Teleostei: Scianidae). Ovogênesis. Folículos Post-Ovulatorios. Atresia. Rev. Brasil. Biol. 50 (2): 523-536.

Recebido em 19.XI.2000; aceito em 24.I.2002. 